\title{
FORMAÇÃO EM GESTÃO PARA APOIO À REFORMA DA ATENÇÃO PRIMÁRIA À SAÚDE EM PORTUGAL E PAÍSES AFRICANOS LUSÓFONOS
}

\author{
FORMATION IN MANAGEMENT FOR SUPPORTING HEALTH REFORMS \\ IN PORTUGAL AND PORTUGUESE-SPEAKING COUNTRIES
}

\begin{abstract}
Luís Velez Lapão ${ }^{1}$ (iD) (0000-0003-0506-1294), Gilles Dussault² (iD (0000-0002-5976-3454)
${ }^{1}$ Universidade Nova de Lisboa, Instituto de Higiene e Medicina Tropical, Global Health and Tropical Medicine, Lisboa, Portugal. <luis.lapao@ihmt.unl.pt>

${ }^{2}$ Universidade Nova de Lisboa, Instituto de Higiene e Medicina Tropical, Lisboa, Portugal.
\end{abstract}

Resumo Apresentamos lições que resultaram de atividades de capacitação dos gestores conduzidas em Portugal no contexto da reforma da atenção primária em saúde e nos países africanos de língua oficial portuguesa, em termos do planejamento e da gestão dos serviços hospitalares e de saúde pública. Descrevemos três programas de formação-ação realizados pela Unidade de Saúde Pública Internacional do Instituto de Higiene e Medicina Tropical de Lisboa, com o apoio de parceiros portugueses e internacionais como a Organização Mundial da Saúde e o Instituto de Medicina Social da Universidade do Estado do Rio de Janeiro. Os programas foram desenvolvidos na base da identificação das necessidades de competências dos participantes e focaram a resolução de problemas concretos com o objetivo de ajudar os gestores a enfrentar as dificuldades inerentes aos processos de reforma. Apesar do seu valor intrínseco, por si só não se mostram suficientes, uma vez que são sempre necessários outros mecanismos, como o acompanhamento continuado dos gestores, sistemas de incentivos coerentes com os objetivos das reformas, ferramentas e recursos (financiamento, sistemas de informação, pessoal qualificado suficiente) adequados para implementar as mudanças. Além disso, a sustentabilidade das intervenções de fortalecimento das capacidades carece de apoio continuado dos decisores políticos.

Palavras-chave recursos humanos em saúde; fortalecimento de competências; reforma em saúde; liderança; Portugal; Palop.
Abstract We present lessons that have resulted from formation activities of managers conducted in Portugal in the context of primary health care reform and in the Portuguese-speaking African countries, regarding planning and management of hospital services. and public health. We describe three action-formation programs conducted by the International Public Health Unit of the Institute of Hygiene and Tropical Medicine-Lisbon, with the support of Portuguese and international partners such as the World Health Organization, and the Institute of Social Medicine of Universidade do Estado do Rio de Janeiro. The programs were developed on the basis of identifying participants' skills needs and focused on solving concrete problems in order to help managers address the difficulties inherent in reform processes. Despite their intrinsic value, they are not sufficient by themselves, as other mechanisms such as the continued monitoring of managers, incentive systems consistent with the objectives of reforms, tools and resources (funding, information systems, qualified staff) are always needed and sufficient to implement the changes. In addition, the sustainability of capacity-building interventions needs continued support from policy makers.

Keywords human resources for health; capacity strengthening; healthcare reform; leadership; Portugal; PALOP. 


\section{Introdução}

O conjunto de instituições prestadoras de serviços de saúde se relacionam entre si para responder às necessidades de saúde das populações. Existe uma inter-relação entre os componentes, não podendo o sistema ser reduzido à análise separada desses componentes (Von Bertalanffy, 1969). Em alguns países, o sistema de saúde evoluiu de forma menos planejada, enquanto em outros houve esforços concertados pelos governos e outros parceiros para desenvolver serviços ajustados às necessidades das populações (World Health Organization, 2000). Duckett (2004) propôs ainda uma abordagem bidimensional para análise de sistemas de saúde: numa dimensão a qualidade, a eficiência e a adequabilidade; na outra, a equidade (Liverpool, 2009).

No contexto europeu e no dos Países Africanos de Língua Oficial Portuguesa (Palops)-Angola, Cabo Verde, Guiné-Bissau, Moçambique, São Tomé e Príncipe -, as reformas do setor da saúde têm formalmente como objetivo a melhoria da equidade de acesso a serviços de qualidade, produzidos de modo eficiente. Entre as diversas estratégias utilizadas, as principais são o fortalecimento da atenção primária em saúde (APS), a melhor integração dos serviços e a adoção de novas medidas para a mobilização mais adequada de recursos financeiros e humanos e para sua distribuição mais equilibrada (Organização Mundial da Saúde, 2008, 2010; World Health Organization, 2010). Recentemente, a Assembleia da Organização das Nações Unidas aderiu ao ambicioso projeto de 'cobertura universal em saúde', ou seja, ao compromisso dos países membros de dar acesso à população a serviços de saúde de qualidade, com base nas necessidades e a custos aceitáveis (United Nations, 2012). Em 2015, a Organização Mundial da Saúde (OMS) veio propor a renovação da estratégia global para os recursos humanos da saúde focada na necessidade de dotar os sistemas de saúde com os recursos adequados para atingir a almejada cobertura universal à saúde (World Health Organization, 2015). Não é possível promover a cobertura universal sem que o sistema tenha os recursos humanos suficientes e adequadamente capacitados.

Nesse contexto, se pode considerar esses objetivos como 'reformas', pois o seu alcance exige mudanças profundas nos diversos níveis do sistema de serviços de saúde. A descentralização da gestão e novas modalidades de prestação de serviços, de regulação ou formação dos prestadores são exemplo disso. No entanto, o sucesso dessas mudanças depende da existência de um ambiente institucional e organizacional que crie condições favoráveis para sua implementação. Em âmbito institucional, é fundamental um quadro jurídico-legal que elimine os obstáculos ao processo de mudança e o facilite mesmo. Isso pode requerer a adoção de legislação que redefina as responsabilidades e as modalidades da tomada de decisão entre os diferentes níveis de governo, a revisão das definições legais das tarefas que venham a ser executadas pelos 
diversos grupos ocupacionais, ou a criação de sistemas de incentivos financeiros e profissionais para estimular a cooperação dos prestadores. Em âmbito organizacional, devem existir capacidades humanas e técnicas de gestão, tais como uma equipe de dirigentes competentes; o acesso a sistemas de informação ágeis e confiáveis, e a instrumentos de gestão adaptados, além da autonomia na distribuição dos recursos.

Os mecanismos institucionais e organizacionais são condicionantes essenciais do sucesso das reformas em saúde, mas não suficientes (Lapão e Pisco, 2019). Na realidade, são os indivíduos, com suas competências, seus valores, sua motivação e dedicação que podem fazer a diferença em cada nível da formulação das políticas, do planejamento, da gestão e sobretudo na prestação de serviços. As novas tarefas correspondentes a novas necessidades dos cidadãos exigem nem sempre somente novas competências, mas também novas modalidades de aquisição de tais competências (Frenk et al., 2010; Barnett et al., 2011).

Sem diminuir a importância fulcral do papel dos prestadores de serviços, pretende-se compreender o comportamento do grupo dos dirigentes (gestores, chefias médicas etc.) e o desafio do reforço da sua capacitação para lidar com um processo de reforma. É um desafio particular para esses profissionais, que devem intervir se confrontando com um contexto complexo, que muda continuamente e de forma frequentemente pouco previsível. Ao contrário dos profissionais da área clínica, os gestores não podem contar, para orientar as suas decisões, com best practices baseadas em evidência científica e replicáveis em qualquer contexto. Existem, contudo, good practices, como exemplos de práticas de gestão conhecidas por favorecer o processo de reforma, mas sempre ligadas a um contexto e a um momento particular na vida de um sistema de serviços, pelo que muitas vezes não são replicáveis (Lapão e Pisco, 2019). Assim, o desafio consiste fundamentalmente na criação, no fortalecimento e na atualização de competências relevantes para dotar os gestores das capacidades para que possam lidar com as exigências da transformação dos processos de produção e de utilização dos serviços de saúde.

Neste artigo, como resultado, se apresentam as lições que resultam de atividades de capacitação conduzidas em Portugal no contexto da reforma da APS e nos Palops, tendo presente que estas se realizaram no contexto da implementação de políticas de fortalecimento da capacidade no âmbito do planejamento, de gestão dos hospitais e dos serviços de saúde pública.

\section{Intervenções para fortalecimento de capacitação}

Uma 'intervenção' é um conjunto de atividades interligadas, com o propósito de atuar sobre uma organização para que comportamentos, processos ou práticas se alterem (Pedersen et al., 2013). O fortalecimento de capacitação exige um conjunto de atividades prévias e durante o processo para garantir o alinhamento com os objetivos e com a motivação dos profissionais. 
Para melhor enquadrar a temática, se vai focar na análise de três programas de fortalecimento de capacitação na área da gestão dos serviços de saúde: o programa FORGEST (formação de FORmadores em GESTão de recursos humanos em saúde); o Programa Avançado de Gestão para Directores Clínicos e Chefias Médicas Hospitalares (PADirH), focado na gestão de departamentos clínicos para dirigentes hospitalares, ambos dirigidos a gestores dos Palops; e o programa PACES, direcionado para gestores e membros de conselhos clínicos dos agrupamentos de centros de saúde (ACESs), uma nova estrutura introduzida em 2009 em Portugal para facilitar a coordenação e a colaboração na rede de atenção de cuidados primários (Lapão e Pisco, 2019).

A análise de cada um dos programas contempla a identificação das necessidades a satisfazer, a abordagem pedagógica escolhida, o desenvolvimento da intervenção e os resultados observados.

\section{FORGEST}

O FORGEST designa um programa de aprendizagem organizado pela Associação para o Desenvolvimento da Medicina Tropical (ADMT) em colaboração com a Unidade de Saúde Pública Internacional do Instituto de Higiene e Medicina Tropical (IHMT), da Universidade Nova de Lisboa; a OMS e o Instituto de Medicina Social da Universidade do Estado do Rio de Janeiro (IMS-Uerj). Este programa avançado foi desenvolvido em resposta às necessidades identificadas pelos dirigentes da saúde nos Palops e em Timor-Leste, no âmbito do Projecto de Apoio ao Desenvolvimento dos Recursos Humanos de Saúde nos Palops (PADRHS-Palop), financiado pela União Europeia e administrado pela OMS. Foi um programa de pós-graduação inovador destinado a futuros formadores e gestores no campo do desenvolvimento dos recursos humanos em saúde (RHS), e que visou apoiar a formação de equipes nacionais de formadores e supervisores em planejamento e gestão em RHS, em que se incluem os sistemas de informação como área-chave para gestão e planejamento. Neste texto, analisa-se a situação da primeira implementação do FORGEST.

Este programa interdisciplinar se destinou a profissionais do setor da saúde e social num contexto do planejamento e gestão de RHS. O programa teve como principal objetivo dotar os profissionais com competências de formadores, capacitá-los no planejamento e na dinamização de ações de formação, e implementar projetos e programas relacionados com o desenvolvimento de RHS. O programa corresponde à frequência da componente curricular do curso de mestrado em saúde pública e desenvolvimento do IHMT, na especialização em políticas e programas. Os conteúdos foram adaptados às necessidades específicas dos participantes e ao contexto dos Palops, dando a possibilidade aos participantes que, após terminarem, continuem os seus estudos para dissertação de mestrado. Como se trata de 'formação de forma- 
dores', o programa incluiu como trabalho prático a preparação de um 'plano de formação' para gestores de RH, em âmbito nacional e em colaboração com os respetivos departamentos de RHS.

O programa teve a duração de nove meses (outubro de 2010 a junho de 2011) e contemplou três módulos presenciais, de duas semanas cada (duas sessões em Cabo Verde e uma em Lisboa), e 12 sessões quinzenais de videoconferência, apoiadas por e-learning. A estrutura curricular contemplou quatro blocos: comunicação em saúde - gestão da formação, promoção e educação para a saúde; métodos em saúde pública - bioestatística, métodos de investigação e epidemiologia; gestão de RHS - políticas, planejamento, gestão e desenvolvimento de RHS; e gestão em serviços de saúde - gestão de projetos e programas em saúde.

A estratégia pedagógica utilizou métodos de trabalho múltiplos que favoreciam o desenvolvimento da autonomia de aprendizagem: aulas participativas, trabalhos de pesquisa e de resolução de problemas individuais e em grupo, bem como leituras e apresentações públicas. Incluiu sessões em videoconferência para debater temas específicos e apoiar o processo de aprendizagem. A avaliação do desempenho dos participantes se fez de acordo com cada unidade curricular (UC), contemplando a dimensão contínua e um projeto final de planejamento de formação em RHS, para ser implementado em cada país. A formação em Cabo Verde teve, durante duas semanas, apoio logístico do Centro de Formação Médica Especializada da Comunidade dos Países de Língua Portuguesa (CPLP), tendo sido o primeiro curso a se realizar nessas instalações. Foi desenvolvido um portal $w e b$, de acesso restrito, com base num Google site, para integrar e armazenar todos os documentos e materiais de apoio pedagógico do curso. Durante a primeira fase, em Cabo Verde, foi ainda garantido que todos os participantes tivessem acesso a e-mail.

A seleção dos candidatos se fez em duas fases. Na primeira, uma vez que o programa se enquadrou no PADRHS, passou pela indicação de profissionais, por parte dos vários ministérios da saúde dos Palops. Numa segunda fase, o IHMT procedeu à avaliação dos requisitos para a frequência do curso: posse de bacharelado ou licenciatura, ser do quadro de nível médio ou superior e possuir experiência ou ligação com atividades de gestão de saúde, sobretudo na área dos RHS.

A 'primeira fase' do curso Forgest considerou os seguintes aspectos: enquadramento dos participantes, provenientes de vários países e com níveis de domínio da língua portuguesa distintos; iniciação do curso com as UCs fundamentais para o desenvolvimento do programa, com ênfase para a área dos RHS e gestão de informação em RHS; preparação dos participantes para utilização do e-mail e do portal web, onde se disponibilizaram documentos e apresentações das várias UCs. Nessa fase, houve um reforço do relacionamento com o Departamento de Recursos Humanos para Saúde da OMS e com o IMS-Uerj no ajustamento do curso. 
Nos quatro meses seguintes, o programa realizou cinco sessões de acompanhamento em videoconferência, apoiadas pela leitura de documentos de apoio (acessíveis no portal web). Todos os países africanos participaram, alguns ocasionalmente com problemas técnicos de comunicação. Somente Timor-Leste nunca conseguiu estabelecer ligação. Assim, optou-se pela disponibilização de um acesso à gravação digital da aula permitindo a sua visualização posterior. Esta 'segunda fase', que ocorreu em Lisboa, permitiu que os alunos aproveitassem para interagir com o corpo docente do IHMT, de modo a iniciar o trabalho do 'Roteiro para a Formação de RHS em Saúde'. Foi igualmente útil para que os alunos começassem a refletir sobre potenciais temas de tese de mestrado, para aqueles interessados em continuar com os estudos. Essa 'fase intercalar' teve os seguintes objetivos: avaliar os primeiros quatro meses de trabalho; continuar com o programa de trabalho e com exercícios práticos associados especificamente à gestão de RHS; formar os alunos na utilização do Moodle, portal da Universidade Nova de Lisboa, onde se disponibilizam os documentos; e iniciar o processo de avaliação da UC e dos seus docentes integrados no processo de qualidade do IHMT.

No período seguinte, entre março e junho, realizaram-se sete sessões em videoconferência, sustentadas ainda pela leitura de documentos de apoio. Assim como na primeira fase, todos os países participaram, alguns ocasionalmente com problemas técnicos de comunicação.

A 'fase final' teve os seguintes objetivos: concluir as UCs iniciadas anteriormente, com ênfase para a área da gestão de projetos e da qualidade; dar continuidade ao processo de avaliação da qualidade do curso; e ainda apoiar os participantes no desenvolvimento dos ‘Roteiros de Formação e Desenvolvimento de RH da Saúde'.

O curso terminou com a organização do 'Seminário Regional dos RHS da Saúde', realizado no Instituto Camões em Cabo Verde, em que os participantes, organizados por países, apresentaram e discutiram os 'Planos de Formação e Desenvolvimento de RHS para a Saúde', de acordo com as necessidades de cada país.

Essa primeira edição do FORGEST permitiu formar um conjunto de 22 profissionais (seis mulheres e 16 homens, com idades compreendidas entre 26 e 55 anos), oriundos de seis países, destacando-se a presença de vários diretores nacionais de RHS, um diretor de escola nacional de saúde pública e vários coordenadores regionais de RHS. Um subproduto do programa é o portal web FORGEST, que continua ativo e serve como fonte de informação e plataforma de comunicação entre os participantes. A boa integração dos vários parceiros institucionais permitiu desenvolver um programa de melhor qualidade e mais adaptado às necessidades dos participantes, levando-os a produzir trabalhos mais relevantes. 
O FORGEST usou a estrutura do mestrado em saúde pública e desenvolvimento, conseguindo manter a qualidade, como comprovaram as avaliações dos alunos, pois foi o primeiro curso a ser avaliado segundo as novas normas da Agência de Avaliação e Acreditação do Ensino Superior (A3ES). O seu caráter inovador beneficiou da colaboração do IHMT com a OMS e a Uerj, por meio da qual se procurou construir uma resposta às necessidades identificadas pelos diversos dirigentes da saúde nos Palops para a área de RHS. O sucesso da formação deveu-se também ao cuidado na programação das ações, com datas bem definidas e cumpridas na íntegra, o que permitiu a organização antecipada da vida profissional e pessoal de todos os envolvidos.

Dos 22 alunos, 11 continuaram os seus estudos para mestrado e um seguiu para doutoramento, tendo dez (quatro de Moçambique, três de Guiné-Bissau, um de São Tomé e Príncipe, um de Angola e um de Cabo Verde) destes já desfendido as suas teses, e atualmente desempenham funções mais destacadas nos respectivos países (Quadro 1).

\section{Quadro 1}

Temas de tese desenvolvidas no âmbito do programa de formadores em gestão de recursos humanos em saúde - FORGEST.

\begin{tabular}{|c|c|c|c|}
\hline Áreas temáticas & \multicolumn{3}{|c|}{ Temas de teses desenvolvidas } \\
\hline $\begin{array}{l}\text { Gestão de recursos } \\
\text { humanos em saúde }\end{array}$ & $\begin{array}{l}\text { Comparação das } \\
\text { expectativas dos alunos de } \\
\text { nível técnico e superior dos } \\
\text { cursos de enfermagem na } \\
\text { Guiné-Bissau }\end{array}$ & $\begin{array}{l}\text { Análise da gestão de } \\
\text { carreiras profissionais } \\
\text { na Direcção Provincial } \\
\text { de Saúde da Zambézia: } \\
\text { contributos para a melhoria } \\
\text { do processo das promoções } \\
\text { e progressões }\end{array}$ & $\begin{array}{l}\text { Supervisão integrada e } \\
\text { formativa no Ministério } \\
\text { da Saúde da Guiné-Bissau } \\
\text { como mecanismos de } \\
\text { reforço do desempenho } \\
\text { dos centros de saúde } \\
\text { das regiões sanitárias de } \\
\text { Bafata, Cacheu e Gabu }\end{array}$ \\
\hline Formação & $\begin{array}{l}\text { Implementação da } \\
\text { formação permanente em } \\
\text { hospitais centrais e gerais } \\
\text { de Angola }\end{array}$ & $\begin{array}{l}\text { Investimento na formação e } \\
\text { na capacitação dos recursos } \\
\text { humanos da saúde em São } \\
\text { Tomé e Príncipe }\end{array}$ & \\
\hline Incentivos/retenção & $\begin{array}{l}\text { O papel dos incentivos } \\
\text { financeiros e não } \\
\text { financeiros para os } \\
\text { enfermeiros de nível básico } \\
\text { e médio a trabalharem } \\
\text { nos centros de saúde da } \\
\text { área do Hospital Geral José } \\
\text { Macano }\end{array}$ & $\begin{array}{l}\text { Motivação e incentivos } \\
\text { para a classe médica } \\
\text { nos hospitais gerais de } \\
\text { Mavalane e José Macamo } \\
\text { na cidade de Maputo - } \\
\text { Moçambique }\end{array}$ & $\begin{array}{l}\text { Retenção de enfermeiros } \\
\text { colocados num hospital } \\
\text { rural de Xinavane e } \\
\text { centros de saúde da } \\
\text { Maluana e Manhiça na } \\
\text { província de Maputo, em } \\
\text { Moçambique }\end{array}$ \\
\hline Tecnologia/informação & $\begin{array}{l}\text { Potencial da telemedicina } \\
\text { na saúde em Cabo Verde: } \\
\text { evacuações sanitárias }\end{array}$ & $\begin{array}{l}\text { Descrição das fontes de } \\
\text { informação sobre recursos } \\
\text { humanos em saúde na } \\
\text { área da saúde sexual e } \\
\text { reprodutiva na Guiné-Bissau }\end{array}$ & \\
\hline
\end{tabular}

Fonte: Os autores. 


\section{PADirH}

O PADirH é um programa de cooperação disponibilizado pelo IHMT, de caráter intensivo de cinco semanas para apoio à governança e gestão de departamentos clínicos hospitalares em contexto africano, dirigido a médicos com cargos de chefia hospitalar ou de direção clínica. O programa é constituído por um componente curricular, um componente de estágio, uma comunidade de prática (e.g. rede de debate e apoio sobre políticas de gestão) e um componente de consultoria e apoio ao desenvolvimento. Este programa é sobretudo enquadrado numa perspetiva de fortalecimento de capacitação, resulta de uma experiência de vários anos e de uma oferta conjunta do IHMT (Universidade Nova de Lisboa), do Centro Hospitalar de Lisboa Centro, do Centro Hospitalar Lisboa Norte, do Hospital Garcia d'Orta, do Hospital de Cascais e do Bureau Regional da OMS/AFRO (Brazzaville), no âmbito do Plano Estratégico de Cooperação em Saúde da CPLP 2009-2012 (PECS-CPLP), contando em algumas edições com financiamento do PADRHS da União Europeia. O objetivo central é a criação de uma equipe de docentes experientes e dedicados no campo da gestão e governança clínica, em contexto hospitalar. As necessidades de formação foram identificadas com base numa análise da literatura sobre as características do hospital africano e sobre a complexidade da gestão em ambiente hospitalar.

Até junho de 2018, realizaram-se sete edições do programa, variando entre 18 e 25 participantes por edição: duas em Angola (Luanda), duas em Cabo Verde (Praia e Mindelo), uma na Guiné-Bissau (Bissau) e outra em Moçambique (Maputo).

Na definição de necessidades, vários aspectos foram considerados:

- 'Programa focado na realidade africana': a organização de uma formação intensiva num país africano tem exigências específicas, pelo que é fundamental a articulação entre as entidades nacionais e locais e a equipe de formadores. Além disso, o hospital africano no espaço lusófono está geralmente instalado em edifício construído antes da independência, e naturalmente já com problemas de instalações (Rypkema e Santing, 1994). Muitos deles sofreram, entretanto, obras que lhes permitem responder melhor às necessidades atuais. Há uma grande heterogeneidade em vários níveis, desde equipamentos aos recursos humanos (Conceição, 2011). Padecem de fragilidades no âmbito de RHS especializados e falta de equipamentos, que muitas vezes são oferecidos, mas carentes da devida manutenção. O 'hospital africano' de primeira referência está geralmente localizado numa região rural com um número médio de camas inferior a duzentos. Esses hospitais apoiam uma população diretamente dependente não só dos centros de saúde, que lhe encaminham doentes mas também de centros de 
saúde em distritos sanitários sem hospital. As equipes clínicas são lideradas por um conjunto de enfermeiros, e os médicos muitas vezes são clínicos gerais (que também fazem cirurgias). Em geral os médicos ficam pouco tempo, o que dificulta toda a gestão e o desempenho do hospital. Tais hospitais referenciam seus doentes para níveis provinciais, regionais ou nacionais ou, ainda, para o estrangeiro. Com referenciação terciária ou quaternária, esses hospitais concentram desproporcionalmente os escassos recursos alocados à saúde.

- 'Problemas típicos de gestão de recursos': o hospital africano oferece usualmente um conjunto de serviços essenciais, como obstetrícia e ginecologia (normalmente associado a uma maternidade e, muitas vezes, a uma residência de espera para grávidas de risco ou em fim de gestação); urgência e cirurgia geral, mas nem sempre presentes (Van Lerberghe et al., 2002); medicina interna; meios de diagnóstico básico (raios X e laboratório de análises clínicas); aprovisionamento de medicamentos e muitas vezes de vacinas (sofrendo ruturas de estoques frequentes (Conceição, Leandro e McCarthy, 2009). Um aspecto preocupante é a taxa de ocupação de camas se situar abaixo de $80 \%-85 \%$ - valores limítrofes geralmente aceitos como indicadores de utilização eficiente das camas hospitalares (Zere et al., 2006). A literatura mostra que existem sérias fragilidades no âmbito do sistema de referenciação. Todos os doentes referenciados não chegam ao hospital, sobretudo por falta de transporte, por taxas e outras despesas, além de fragilidades no atendimento hospitalar (Bossyns e Van Lerberghe, 2004). Os sistemas de triagem são também comumente frágeis.

- 'Desafios da gestão hospitalar': num hospital, os profissionais possuem um elevado nível de qualificação, o que dificulta, e até impossibilita, a supervisão hierárquica tradicional. Sendo o hospital uma organização muito diferenciada, vertical e horizontalmente, a sua produção situa-se sobretudo no centro operacional (emergência, cirurgias, consultas etc.). Nesse caso, os serviços de apoio servem sobretudo para assistir o centro operacional; a sua tecnoestrutura e a hierarquia são embrionárias (fazem parte da arquitetura de apoio) - por exemplo: o enfermeiro diretor e a enfermeira supervisora podem ser considerados como elementos da tecnoestrutura (Monteiro, 1999). As questões de dimensão e de escala são particularmente complexas em estados pequenos (Cabo Verde, Guiné-Bissau e São Tomé e Príncipe) ou insulares (Cabo Verde, São Tomé e Príncipe e o Arquipélago dos Bijagós).

- 'Qualidade e obtenção de ganhos em saúde': a dinâmica dos serviços de saúde, sobretudo no contexto hospitalar, impõe que qualquer chefia hospitalar deve estar munida de fortes competências na área da gestão, liderança e da governança clínica (e.g. implementação e uso adequado dos protocolos de perscrição de antibióticos). A gestão hospitalar deve tam- 
bém promover o bom uso de instrumentos (planejamento, avaliação etc.) que permitam apoiar a decisão sobre a alocação de recursos (RH, tecnologia, financeiros etc.) alinhada com os objetivos da organização. Cada vez mais se enfoca na relação custo-efetividade, na segurança dos doentes, na sustentabilidade e na qualidade, com uma preocupação crescente de reforçar o acesso e a universalidade. A governança clínica define um quadro de referência para atuação dos profissionais no processo decisório, e depende profundamente de acesso a informação clínica que suporte essas decisões (Som, 2004). A governança clínica deve estar orientada para o hospital na sua globalidade, enquanto a gestão clínica visa a setores clínicos específicos no hospital, isto é, as principais especialidades hospitalares (medicina interna, pediatria, cirurgia geral, obstetrícia e ginecologia etc.) e os seus diversos setores (internamento, hospital de dia, ambulatório, urgências etc.). Na gestão clínica se cruzam aspectos de gestão corrente de recursos, que até são os mais dispendiosos no contexto hospitalar, com um conhecimento científico muito diferenciado e uma ética (personalizada e institucionalizada, daí a importância crescente das comissões de ética) bem definida e aceita pela sociedade em geral, com forte orientação para o doente, as suas necessidades e o seu bem-estar, e o seu contexto social mais imediato.

- 'Desenvolvimento organizacional dos profissionais de saúde': o esforço de reorganização começa a se manifestar por um conjunto de tentativas de mudança (Lapão, 2005) e integração dos serviços (Meliones, 2000). Tais transformações necessitam de mudanças na forma de trabalhar da organização, desde a reorganização dos processos de produção (por exemplo, equipes multidisciplinares), passando pela utilização de meios como a internet, até à reorganização dos serviços de apoio, que exigem capacidade de gestão da mudança e de desenvolvimento organizacional dos profissionais. $\mathrm{O}$ desenvolvimento organizacional é a forma como os vários colaboradores da organização se relacionam entre si, e como podem melhorar esta interação para aprimorar os serviços prestados. Daí a necessidade crescente de que as lideranças profissionais se afirmem cada vez mais na definição da visão e da missão dos hospitais e na consecução dos objetivos que delas resultam. Por sua vez, os serviços de saúde devem ser enquadrados numa lógica de serviços, isto é, as organizações de saúde devem fornecer serviços que deem resposta às 'necessidades' dos indivíduos e das populações, criando 'canais' de acesso adequados (consultas, emergência, cirurgias etc.), com serviços definidos de acordo com as regras da 'governança clínica'. Ao se partir dessa lógica, consegue-se determinar as necessidades de 'recursos', incluindo RHS, para que os serviços sejam prestados adequadamente.

- 'Gestão da interface com a atenção primária': o desenvolvimento hospitalar não pode ignorar a interface com a APS. A governança clínica dos hospitais 
têm grande dependência das interfaces com a APS, entre setores privado e público, por meio da integração dos serviços de que as redes de referenciação e a contratualização são um elemento fundamental. No ideal, para que a integração entre hospitais e APS dê frutos, são necessárias pelo menos quatro condições (Ramsey, Fullop e Edwards, 2009): um modelo de governança que alinhe as atividades dos cuidados primários e secundários; a existência de um sistema de informação que permita a partilha de registros clínicos que apoiem a gestão e o desempenho dos profissionais; a coordenação dos cuidados (gestão de recursos, sistemas de informação etc.) e a governança clínica (Shortell e Schmittdiel, 2004), para ser possível prestar cuidados de forma mais econômica e com maior qualidade - uma organização integrada poderá desenvolver um tipo de planejamento que operadores desagregados não podem (Bindman et al., 2005); e a capacidade para potenciar melhorias de eficiência (Newhouse, 1994).

Os objetivos da aprendizagem do PADirH são baseados nas considerações dos parágrafos anteriores: rever e reforçar conhecimentos gerais de gestão com particular ênfase na liderança, na valorização da inovação organizativa e nas técnicas de gestão mais relevantes para o contexto hospitalar; contextualizar a importância da gestão hospitalar no sistema de saúde, levando em consideração as características do país (World Health Organization, 2008); compreender o conceito de governança e de gestão clínica nas principais especialidades hospitalares (medicina interna, cirurgia geral, pediatria, obstetrícia e ginecologia); desenvolver a orientação para o cliente, para ganhos em saúde e para melhorar o acesso aos serviços. De modo a apoiar essa aprendizagem, desenvolveu-se um quadro de referência, orientador do que são as necessidades temáticas de aprendizagem para chefias médicas hospitalares para melhor assumirem a direção clínica dos serviços clínicos hospitalares (IHMT, 2012).

O quadro de referência tem como base de incidência o contexto do país (economia, política, cultura e sociedade) e o sistema de saúde onde se situa o hospital. A esse quadro corresponde um conjunto de aspectos dominantes para o desenvolvimento de atividades diárias (operacionais e táticas) e periódicas (táticas e estratégicas) às quais está associado um conjunto de competências como referência (Figura 1). 
Competências de referência do PADirH (Programa Avançado de Gestão para Diretores Clínicos e Chefias Médicas Hospitalares)

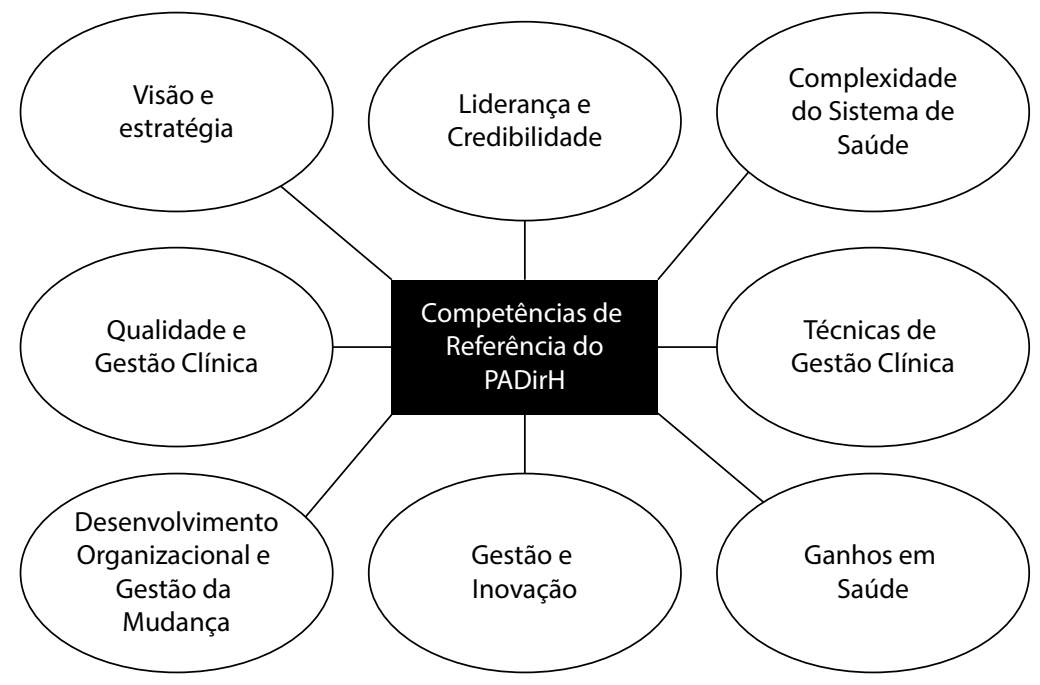

Fonte: Os autores.

Esse quadro de referência visa enquadrar o papel da chefia médica hospitalar nas suas diversas vertentes, no contexto específico do serviço que dirige, ou vai dirigir, e da interação com os serviços que o vão apoiar. Assim, a aprendizagem pode se refletir em termos de aquisição de elementos de conhecimento, desenvolvimento de competências e reforço e adoção de determinadas atitudes (Prestwich e Ho-Kim, 2007). A estas três dimensões acrescentam-se duas outras: os 'valores', pessoais e de referência das organizações no seu contexto social; e os 'comportamentos' que se refletem no cotidiano e em situações concretas.

A avaliação do programa inclui quer a avaliação da aprendizagem dos alunos, quer a do ensino que lhes foi oferecido. A avaliação da aprendizagem inclui cinco elementos: inquérito hospitalar; pré-teste; seminários dos alunos; espaço projeto; e exame final. Este processo de validação do ensino tem a finalidade de perceber como foi a formação percepcionada pelos participantes e introduzir melhoramentos nas edições seguintes do PADirH.

Um dos efeitos mais significativos do programa resulta do apoio a projetos de reorganização interna que sejam úteis ao hospital. Estes são desenvolvidos ao longo do programa por meio do 'aprender fazendo' (learning-by-doing) e de aproveitar as interações e os conhecimentos técnicos dos diversos docentes. A aposta na 'gestão de projetos' como ferramenta de referência para apoiar a gestão da mudança, com a implementação e o uso eficiente dos recursos (Turner e Keegan, 2001; Lapão e Dussault, 2011), permite ainda contribuir 
para o alinhamento da escolha do projeto com os objetivos estratégicos. Essa é uma oportunidade de testar o potencial da gestão por objetivos focada nos resultados (Kaplan e Norton, 2000). Para ter impacto, o 'espaço de projeto' foi estruturado em redor do diagnóstico dos problemas (mais significativos) de qualidade do hospital (e para cada equipe). Assim, cada uma das equipes desenha e planeja um 'projeto de mudança organizacional', no âmbito da 'segurança do paciente', a ser apresentado no final do curso. A escolha da temática se baseia no 'sistema de ideias' (Hamel, 2006), com o intuito pedagógico duplo de gerar ideias para resolver problemas e ao mesmo tempo garantir o envolvimento no processo de escolha e consequentemente na implementação (Lines et al., 2005). Esse problema é trabalhado pela equipe, na forma de projeto, em quatro semanas. Ao longo das várias edições, se tem verificado surgirem projetos nas áreas de reorganização do bloco operatório, reorganização dos serviços de emergência, reorganização das maternidades, criação de comissões de infecção etc., com claro impacto na melhoria dos serviços.

Por sua vez, os seminários permitiram que os participantes desenvolvessem outros assuntos importantes para a gestão clínica dos hospitais. A sua preparação e apresentação de seminário é uma técnica de aprendizagem que inclui pesquisa, planejamento, apresentação, discussão e debate. O trabalho de discussão em grupo não é mais do que uma participação social, uma oportunidade de estudar e debater um problema, avaliar ideias novas, tomar uma decisão comum. Trata-se de uma atividade cooperativa para a solução de um problema (problem-solving) que procura o consenso do grupo. Ao longo das diversas edições, os temas mais desenvolvidos têm sido 'gestão de $\mathrm{RH}^{\prime}$, 'gestão de informação para apoio à governança clínica', 'gestão da interface entre atenção primária e hospitais' e 'gestão farmacêutica no hospital'. Como resultado não previsto inicialmente do programa, vários dos participantes vêm assumindo novas responsabilidades nos seus hospitais, e alguns têm prosseguido com os estudos.

\section{PACES}

A reforma dos serviços de saúde, no sentido de reforçar a atenção primária e proporcionar a cobertura universal, começou efetivamente em 1971, com a criação de centros de saúde. Em 2008, o governo criou uma rede de 73 agrupamentos de centros de saúde (ACESs), abrangendo, cada um, populações que variam de cinquenta mil a duzentas mil pessoas. Esses agrupamentos incluem centros de saúde tradicionais, bem como as mais inovadoras unidades de saúde familiar (USFs). Em abril de 2013, existiam 353 USFs, abrangendo uma população de 4.345 .000 pessoas, o que representa cerca de $50 \%$ dos abrangidos pelo Serviço Nacional de Saúde (SNS) em Portugal, empregando 2.452 médicos e 2.428 enfermeiros (Portugal, 2013). Os novos ACESs são 
uma estrutura administrativa encarregada de melhorar a coordenação e a eficiência dos serviços de saúde em âmbito local por meio de planejamento e compartilhamento de recursos (Lapão e Pisco, 2019). Cada ACES é gerenciado por uma equipe composta por um diretor executivo, um conselho clínico de quatro membros, que representam os diferentes grupos profissionais, e uma unidade de apoio à gestão. ACESs são apenas parcialmente autônomos, pois eles não têm autonomia financeira; dependem de uma administração regional de saúde (ARS) que tem a responsabilidade de planejamento e alocação de recursos (i.e. semelhante a uma Secretaria de Saúde estadual). No entanto, os ACESs representam um passo significativo no sentido da descentralização em um país que tem uma longa tradição de centralização decisória. A sua criação suscitou a questão do fortalecimento da capacitação de gestão para liderar equipes multiprofissionais e de como irão se ajustar a um novo modelo de governança concebido para oferecer serviços adaptados às necessidades específicas de uma população. Foram criadas, para essa finalidade, cerca de quatrocentas posições de gestão com forte componente de liderança, mas a maioria dos nomeados não tinha qualquer experiência gerencial.

Para reforçar as competências de gestão e de liderança adaptadas às necessidades desses novos gestores se desenvolveu uma estratégia de formação específica. A implementação da reforma organizacional é, por definição, a passagem de um modo de fazer as coisas para outro, e é um processo multidimensional em que as capacidades para efetuar a mudança são necessárias em vários níveis (Braithwaite, 2008; Dickson, 2009). A mais óbvia é a de indivíduos que podem precisar de dominar novas competências. Os gestores formais podem ser os primeiros agentes de mudança, mas também podem falhar se não tiverem as habilidades certas ou práticas.

O Ministério da Saúde de Portugal escolheu como estratégia para aumentar a capacidade dos futuros gestores dos ACESs um programa de formação-ação de um ano. O programa foi concebido com o argumento de que o novo tipo de organização necessitaria de "uma nova cultura de gestão e liderança, com base em valores centrados no cidadão e confiança profissional e empreendedorismo" (Portugal, 2007, pag.11). A Missão Cuidados Primários (MCSP) foi encarregada de "criar programas de treinamento para desenvolver competências-chave de gestão e liderança" e de "promover a identificação de gestão e liderança de melhores práticas e de partilha de experiências" entre os diretores (Portugal, 2007, p. 34). O PACES foi desenvolvido por uma equipe da MCSP com a colaboração de um docente do IHMT. A fase de concepção levou cerca de um ano, anterior à seleção dos futuros gestores. A formação começou imediatamente depois da sua nomeação, em dezembro de 2008. Dos 73 nomeados, $80 \%$ eram médicos, $15 \%$ gestores ou economistas, e $5 \%$ eram de outras profissões de saúde; quatro de cinco tinham cinquenta anos ou mais, e as mulheres representaram pouco mais de $40 \%$ dos participantes. 
Outro programa PACES foi desenvolvido um ano mais tarde para incluir os membros dos conselhos clínicos, cerca de trezentas pessoas, de maio de 2009 a maio de 2010.

O desenho do programa levou em consideração a lei que define as regras de seleção para diretores executivos e a obrigação da MCSP de desenvolver um 'programa de formação em gestão e em liderança para executivos dos ACESs, como parte do Plano Estratégico do Ministério da Saúde (Portugal, 2007). O nome PACES foi escolhido deliberadamente para indicar a ideia de 'ritmo' para a mudança. A concepção do programa PACES baseou-se nas seguintes premissas e pressupostos: a complexidade do desenvolvimento da reforma da atenção primária, que requer uma forte liderança (Plsek e Wilson, 2001; Lapão, 2005; Starfield, 2011) e programas mais longos de formação (Bass, 1990; Alimo-Metcalfe e Lawler, 2001); a importância da formação-ação, numa lógica de metodologia ativa (Lapão, 2010), com foco na resolução de problemas (Mintzberg, 2006), especificamente para responder ao desenvolvimento dos ACESs e às necessidades de implementação (Gomes e Lapão, 2009); a necessidade de uma perspectiva de gestão da inovação (Hamel, 2006) para lidar com os obstáculos burocráticos do atual sistema de saúde; a necessidade de métodos de formação adaptados aos gestores, especialmente para o desenvolvimento de habilidades cognitivas e interpessoais (Arthur Jr. et al., 2003; Collins e Holton, 2004; Alimo-Metcalfe e Alban-Metcalfe, 2005); o foco na 'gestão de projetos' como a ferramenta-chave para lidar com a implementação da mudança e o uso eficiente dos recursos (Turner e Keegan, 2001); a importância do desenvolvimento de uma rede de pares para compartilhar os sucessos de gestão como 'boas práticas' (Dyer e Nobeoka, 2000; Hamel, 2006); e a importância de expor os participantes ao melhor conhecimento disponível em gestão (Farmer e Chesson, 2001).

Os objetivos do programa foram definidos em termos de aquisição de elementos de conhecimento, desenvolvimento de competências e adoção ou fortalecimento de atitudes. A capacitação em gestão do projeto foi um objetivo principal e estruturou-se em torno do diagnóstico dos principais problemas reais dos ACESs. Pretendeu-se que trabalhassem a concepção e o planejamento de um 'projeto de mudança organizacional'. Os participantes foram convidados a selecionar um problema, baseado no 'sistema de ideias' (Hamel, 2006), e depois em grupo negociar e escolher um único problema que seria objeto de trabalho do grupo durante a duração do programa (um ano). O progresso foi monitorado e discutido a cada cinco semanas.

Relativamente à formação, os vários docentes foram convidados a adotar estratégias pedagógicas que levassem os participantes a resolver problemas esperados de serem encontrados na vida real, como a implementação do regulamento do ACES, planejamento de recursos humanos, negociação com os coordenadores de USFs e definição de objetivos e metas das unidades 
funcionais. Essa escolha baseou-se na suposição de que formação-ação é uma ferramenta para o desenvolvimento organizacional; os participantes aprendem por meio da discussão e troca de experiências, pois o trabalho em grupo facilita a análise de problemas e busca de soluções, o que aumenta a curiosidade e as atitudes positivas para com a inovação (Kreitner e Kinicki, 2007). Em relação ao conteúdo, o programa teve em consideração o mestrado internacional em liderança na saúde da Universidade McGill (Gosling e Mintzberg, 2003), que é organizado em torno de cinco dimensões-chave para a formação de profissionais que lidam com gestão de serviços de saúde: a mentalidade catalítica (procurando maneiras de conseguir a mudança); a mentalidade de colaboração (aprender a apreciar relação de trabalho); a mentalidade reflexiva (permitindo a ampliação de perspectivas e oportunidades); a mentalidade mundana (praticar o 'navegar' pelo sistema de saúde); e a mentalidade analítica (compreender os papéis e as principais organizações).

Adicionalmente, foi criada uma plataforma on-line para servir como repositório de documentos e como espaço para colaboração e compartilhamento de resultados. Cada grupo regional (norte, centro e sul) usou-a como sua maneira 'oficial' de comunicação entre si e com as suas agências regionais de saúde (ARSs). Os grupos usaram várias estratégias para avançar com os seus projetos. Os problemas selecionados foram: gestão logística, quadro de avaliação do desempenho de recursos humanos, processo de planejamento estratégico, alteração das atividades de gestão de suporte e serviços de negociação. Após as sessões presenciais, os seis grupos receberam treino tutorial ao longo de todo o resto do processo.

Os projetos desenvolvidos pelos participantes foram apresentados e discutidos num seminário final com a presença do secretário de estado da saúde, responsável pela atenção primária no Ministério da Saúde. Esses projetos, conjuntamente com uma descrição dos 73 ACESs e suas principais realizações durante o programa de treinamento, foram publicados em livro (Lapão, 2010).

Apesar de muitas dificuldades, o PACES criou oportunidades para que os diretores executivos pudessem analisar problemas de gestão e compartilhar soluções potenciais com os colegas. Alguns efeitos positivos podem ser atribuídos ao programa, além dos ganhos de aprendizagem produzidos. O PACES facilitou a criação de um espírito de grupo e hábitos de redes entre os gestores. Em discussão com eles, vários participantes mencionaram que o programa ajudou em suas relações com as autoridades regionais e que o compartilhamento de estratégias sobre como estimular a criação de USFs foi útil. Os gerentes mais jovens ( $<40$ anos) e menos experientes particularmente se beneficiaram da sua participação tanto em termos de aprendizagem como na obtenção de reconhecimento das suas capacidades pelos seus pares (Lapão e Dussault, 2011). 


\section{Lições aprendidas e recomendações}

A experiência de desenvolvimento desses programas de reforço de capacitação em Portugal e nos Palops permite elencar um conjunto de lições relevantes.

O contexto de formação-ação permitiu obter bons resultados dos projetos no terreno. Do ponto de vista teórico, se reconhece a importância de se desenvolverem novas competências por meio da realização de atividades relacionadas com realidade do trabalho, isto é, formação-ação (Lapão e Dussault, 2011), com foco na resolução de problemas reais e concretos (Mintzberg, 2006), e especificamente respondendo às necessidades dos profissionais no contexto da complexidade da saúde (Plsek e Wilson, 2001), concretamente tendo em conta a perspetiva da inovação de gestão (Hamel, 2006), para que se identifiquem e se promovam as condições que permitam encontrar alternativas 'inovadoras', no contexto concreto, de modo a se ultrapassarem os obstáculos usualmente burocráticos e outros existentes de fato -, neste caso, nos ACESs.

É deveras importante fazer o diagnóstico de necessidade e de se disponibilizar tempo suficiente para preparar o programa e alinhá-lo com as necessidades dos formandos. Cada programa deve permitir desenvolver as competências mais necessárias. Uma característica comum aos três programas foi o esforço de envolver previamente os participantes na identificação das suas necessidades de competências e de fazer o diagnóstico das necessidades de formação, para em seguida se construir um programa que desse a resposta adequada a essas necessidades. Num dos casos, PADirH, tem-se ainda aproveitado a avaliação do ensino pelos participantes para proceder à atualização e melhoria do curso.

Cada vez mais a gestão da saúde exige conhecimento multidisciplinar. É por isso importante a utilização de uma variedade de docentes para apoiar uma formação. A diversidade e complexidade das necessidades de formação e o correspondente desafio pedagógico de concretizá-las obrigam a uma integração de conteúdos e da orientação dos diversos intervenientes, focados num resultado final integrado. Entretanto, é necessário que os programas considerem ainda o desenvolvimento de competências na área da inovação.

No caso de formação profissional, é aconselhável o acompanhamento continuado dos departamentos de RHS dos ministérios da Saúde, pois a frequência dos programas de formação-ação em si não é suficiente. Outras capacidades e a adequação dos modelos de governança poderão ser necessárias (por exemplo: descentralização de processos de decisão). Aspectos importantes são a estrutura organizacional e o ambiente institucional, políticas concretas, regras, normas, processos etc. Os sistemas de incentivos são igualmente necessários para ajudar e motivar as pessoas a alcançarem os objetivos das reformas, tanto quanto infraestruturas e outras ferramentas e recursos (financiamento, informações, pessoal etc.). O desafio resulta em projetar e implementar o tipo certo 
de estratégias que contribuam para criar o ambiente adequado para apoiar a mudança efetiva, como alguns autores afirmam: "todo sistema humano - um país, uma rede, um grupo ou indivíduo - tem dentro de si o potencial para desenvolver a sua própria capacidade" (Baser e Morgan, 2008, p. 27). Por isso o desenvolvimento de capacidades é essencialmente um "processo de desbloqueio ou de indução dessa capacidade".

A utilização das tecnologias de informação (da internet, por exemplo) é um apoio fundamental à comunicação e ao desenvolvimento das competências. Os três programas mencionados têm procurado potenciar ao máximo a utilização dessas tecnologias para benefício dos participantes. As tecnologias de informação, as videoconferências, a internet são hoje contributos importantes e suportes de uma pedagogia que permite melhor comunicação entre os participantes e os docentes, bem como o acesso a documentos de apoio. A sua utilização no processo formativo deve inspirar o seu uso no dia a dia.

Por vezes, dada a dimensão específica das formações, podem acontecer atrasos resultantes da complexidade dos processos burocráticos, pelo que convém planejar com tempo.

\section{Conclusões}

O fortalecimento de capacitação é fundamental para criar gestores com competências e atitudes para suportar os desafios consentâneos com os de uma reforma no setor da saúde. O desenho de intervenções de capacitação de dirigentes representa processos complexos e exigentes. A criação e a implementação de programas de formação-ação mostram-se um bom mecanismo para capacitar dirigentes da saúde e ajudá-los a enfrentar as dificuldades inerentes a processos de reforma. Apesar do seu valor intrínseco, por si só não se mostram suficientes, uma vez que são sempre necessários outros mecanismos como o acompanhamento continuado dos gestores, sistemas de incentivos coerentes com os objectivos das reformas e ferramentas e recursos (financiamento, sistemas de informação, pessoal qualificado suficiente) adequados para implementar as mudanças. Além disso, a sustentabilidade das intervenções de fortalecimento das capacidades carece de apoio continuado dos decisores políticos.

\section{Colaboradores}

Ambos os autores colaboraram na organização e abordagem metodológica do artigo. Luís Lapão escreveu a primeira versão do texto e Gilles Dussault foi responsável pela revisão final. 


\section{Financiamento}

Este suplemento "Educação e Trabalho em Saúde: diálogos e experiências no Brasil e em Portugal" foi realizado com apoio da Coordenação de Aperfeiçoamento de Pessoal de Nível Superior (Capes) - Código de Financiamento 001; e do Departamento de Pesquisa em História das Ciências e da Saúde da Casa de Oswaldo Cruz (Depes/COC/Fiocruz, 6151000000). Todos os autores declaram que não há conflito de interesses.

\section{FORMACIÓN EN GESTIÓN PARA APOYO DE LAS REFORMAS SANITARIAS EN PORTUGAL Y EN LOS PAÍSES DE LENGUA OFICIAL PORTUGUESA}

Resumen En ese artículo presentamos lecciones que resultaran de actividades de capacitación de los gestores conducidas en Portugal en el contexto de la reforma de la atención primaria en Salud y en los países africanos de lengua oficial portuguesa a nivel de planeamiento y de la gestión de los servicios hospitalarios y de salud pública. El artículo describe tres programas de formación-acción realizados por la Unidad de Salud Pública Internacional del Instituto de Higiene y Medicina Tropical, con el apoyo de socios portugueses e internacionales como la Organización Mundial de Salud, y el Instituto de Medicina Social, Universidad del Estado del Rio de Janeiro. Los programas se desarrollaron sobre la base de la identificación de las necesidades de competencias de los participantes y enfocaran en la resolución de problemas concretos con el objetivo de ayudar los gestores a enfrentar las dificultades inherentes a los procesos de reformas. A pesar de su valor intrínseco, no son suficientes por sí solas, ya que siempre son necesarios otros mecanismos, como el monitoreo continuo de los gestores, sistemas de incentivos coherentes con los objetivos de las reformas, herramientas y recursos (financiación, sistemas de información, personal cualificado suficiente) adecuados para implementar los cambios. Además, la sostenibilidad de las intervenciones de fortalecimiento de las capacidades carece de apoyo continuado de los responsables políticos. Palabras clave recursos humanos en salud; fortalecimiento de competencias; reforma en salud; liderazgo; Portugal; PALOP. 


\section{Referências}

ALIMO-METCALFE, Beverly; ALBAN-METCALFE, John. Leadership: time for a new direction? Leadership, Newbury Park, v. 1, n. 1, p. 51-71, fev. 2005.

ALIMO-METCALFE, Beverly; LAWLER, John. Leadership development in UK companies at the beginning of the twenty-first century: lessons for the NHS? Journal of Management in Medicine, v. 15, n. 5, p. 387-404, 2001.

ARTHUR Jr, Winfred et al. Effectiveness of training in organizations: a meta-analysis of design and evaluation features. Journal of Applied Psychology, v. 88, p. 234-245, 2003.

BARNETT, Julie et al. Understanding innovators' experiences of barriers and facilitators in implementation and diffusion of healthcare service innovations: a qualitative study. $B M C$ Health Services Research, v. 11, p. 342-351, 2011.

BASER, Heather; MORGAN, Peter. Capacity, performance and results. European Centre for Development Policy Management, 2008. Disponível em: <http://www.ecdpm.org/capacitystudy>. Acesso em: 31 ago. 2019.

BASS, Bernard M. Bass and Stodgill's Handbook of Leadership: theory, research, and applications. New York: The Free Press, 1990.

BINDMAN, Andrew B. et al. The impact of medicaid managed care on hospitalizations for ambulatory care sensitive diagnosis. Health Services Research, [s.l.], v. 40, n. 1, p. 295314, 2005.

BOSSYNS, Paul; VAN LERBERGHE, Wim. The weakest link: competence and prestige as constraints to referral by isolated nurses in rural Niger. Human Resources for Health, [s.l.], v. 2, p.1-8, 2004.

BRAITHWAITE, Jeffrey. $\mathrm{L}(\mathrm{H}) \neq \Sigma\left(\mathrm{m}^{1}, \mathrm{~m}^{2} \ldots\right.$ $\left.\mathrm{m}^{\mathrm{n}}\right)$. Leadership in Health Services, [s.l.], v. 21, n. 1, p. 8-15, 2008
COLLINS, Doris B.; HOLTON, Elwood F. The effectiveness of managerial leadership development programs: a meta-analysis of studies from 1982 to 2001. Human Resource Development Quarterly, [s.1.], v. 15, n. 2, p. 217-248, 2004.

CONCEIÇÃO, Cláudia; LEANDRO, Alexandra; McCARTHY, Mark. Apoio governamental à investigação em saúde pública: um perfil europeu. Revista Portuguesa de Saúde Pública, [s.l.], v. 27, n. 1, p. 71-80, 2009.

CONCEIÇÃO, Maria C. Hospitais de primeira referência, distrito de saúde e estratégia dos cuidados de saúde primários em Moçambique. 357 f. Tese de Doutoramento - Instituto de Higiene e Medicina Tropical, Universidade Nova de Lisboa, Lisboa, 2011.

DICKSON, Graham. Transformations in Canadian health system leadership: an analytical perspective. Leadership in Health Services, [s.1.], v. 22, n. 4, p. 292-305, 2009.

DUCKETT, Stephen. The Australian health care system. Melbourne: Oxford University Press, 2004.

DYER, Jeffrey H.; NOBEOKA, Kataro. Creating and managing a high-performance knowledge-sharing network: the Toyota case. Strategic Management Journal, [s.l.], v.21, n.3, p. 345-367, 2000

FARMER, Jane; CHESSON, Rosemary. Health care management: models for evidence-based practice. Journal of Management in Medicine, [s.l.], v. 15, n. 4, p. 266-284, 2001.

FRENK, Julio et al. Health professionals for a new century: transforming education to strengthen health systems in an interdependent world. The Lancet, [s.l.], v. .376, n. 9756, p. 1.923-1.958, 2010.

GOMES, Pedro; LAPÃO, Luís V. The role of a nation-wide information system in improving the access to surgery in Portugal. Studies in 
Health Technology and Informatics, [s.1.], v.71, p. 978-981, 2009.

GOSLING, Jonathan; MINTZBERG, Henry. The five minds of a manager. Harvard Business Review, Boston, v. 81, n. 11, p. 54-63, 2003.

HAMEL, Gary. O futuro da gestão. Lisboa: Actual Editora, 2006. (Colecção Harvard).

INSTITUTO DE HIGIENE E MEDICINA TROPICAL (IHMT). PADirH: Programa Avançado de Gestão para Diretores Clínicos/Chefias Médicas Hospitalares. Documento interno. Lisboa: Instituto de Higiene e Medicina Tropical, 2012.

KAPLAN, Robert S.; NORTON, David P. The strategy-focused organization: how balanced scorecard companies thrive in the new business environment. Boston: Harvard Business School Press, 2000.

KREITNER, Robert; KINICKI, Angelo. Comportamento organizacional. New York: McGraw-Hill, 2007.

LAPÃO, Luís V. A complexidade da saúde obriga à existência de uma arquitetura de sistemas $\mathrm{e}$ de profissionais altamente qualificados. Revista de Estudos Politécnicos, Barcelos, v. 2, n. 4, p. 15-27, 2005.

LAPÃO, Luís V. PACES: Programa Avançado de Gestão para Directores Executivos dos ACES. Lisboa: INA Editora, 2010.

LAPÃO, Luís V.; DUSSAULT, Gilles. PACES: a national leadership program in support of primary-care reform in Portugal. Leadership in Health Services, [s.1.], v. 24, n. 4, p. 295307, 2011.

LAPÃO, Luís V.; PISCO, Luís. A reforma da atenção primária à saúde em Portugal, 20052018: o futuro e os desafios da maturidade. Cadernos de Saúde Pública, Rio de Janeiro, v.35, n. 14, e00042418, 2019. DOI: http://dx.doi. org/10.1590/0102-311X00042418.
LINES, Rune et al. The production of trust during organizational change. Journal of Change Management, [s.1.], v. 5, n. 2, p. 221-245, 2005.

LIVERPOOL. Health care system, 2009. Disponível em: <liverpool-ha.org.uk>. Acesso em: 31 ago. 2019.

MELIONES, Jon. Saving money, saving lifes. Harvard Business Review, Boston, nov.-dez. 2000 .

MINTZBERG, Henry. Developing leaders? Developing countries? Development in Practice, [s.l.], v. 16, n. 1, p. 4-14, 2006.

MONTEIRO, Ileana P. Hospital, uma organização de profissionais. Análise Psicológica, Lisboa, v. 2, n. 17, p. 317-325, 1999.

NEWHOUSE, Joseph. P. Frontier estimation: how useful a tool for health economics? Jour nal of Health Economics, [s.l.], v. 13, n. 3, p. 317-322, 1994.

ORGANIZAÇÃO MUNDIAL DA SAÚDE (OMS) Cuidados primários de saúde: agora mais que nunca. Relatório Mundial da Saúde 2008. Genebra: Organização Mundial da Saúde, 2008.

ORGANIZAÇÃO MUNDIAL DA SAÙDE (OMS) Financiamento dos serviços de saúde: o caminho para a cobertura universal. Relatório Mundial da Saúde 2010. Genebra: Organização Mundial da Saúde, 2010.

PEDERSEN, Janice S. et al. The puzzle of changing relationships: does changing relationships between healthcare service users and providers improve the quality of care? Londres: Health Foundation, 2013. 64 p. Disponível em: <https:// www.rand.org/pubs/external_publications/ EP51380.html>. Acesso em: 31 ago. 2019.

PORTUGAL. Ministério da Saúde. Missão para os Cuidados de Saúde Primários. MCSP. Reforma dos Cuidados de Saúde Primários Plano Estratégico 2007 - 2009. Lisboa: Ministério da Saúde, 2007. Disponível em: <http://www2. acss.min-saude.pt/Portals/0/Plano_Estrategico_2007_2009.pdf> Acesso em: 31/08/2019 
PORTUGAL. Ministério da Saúde. Missão para os Cuidados de Saúde Primários. Lisboa: Ministério da Saúde, 2013.

PLSEK, Paul E.; WILSON, Tim. Complexity, leadership, and management in healthcare organizations. British Medical Journal, [s.l.], v. 323, n. 7.315, p. 746-749, 2001.

PRESTWICH, Roger; HO-KIM, Thu-Mai. Knowledge, skills and abilities of international business majors. Journal of Teaching in International Business, [s.l.], v. 19, n. 1, p. 29-55, 2007.

RAMSEY, Angus; FULLOP, Naomi; EDWARDS, Nigel. The evidence base for vertical integration in health care. Journal of Integrated Care, London, v. 17, n. 2, p. 3-12, 2009.

RYPKEMA, Steve M.; SANTING, Ruth C. A. The district health system: cooperation between NGO's and health authorities at district level, a Medicus Mundi mail survey. Medicus Mundi Internationalis, Nijmegen, 1994.

SHORTELL, Stephen M.; SCHMITTDIEL, Julie. Prepaid groups and organized delivered systems: promise, performance, and potential. In: Toward a Twenty-First-Century Health Systems. San Francisco: Jossey-Bass, 2004.

SOM, Chandra V. Clinical governance: a fresh look at its definition. Clinical Governance: An International Journal, v. 9, n. 2, p. 87-90, 2004.

STARFIELD, Barbara. Politics, primary healthcare and health. Journal of Epidemiology and Community Health, [s.1.], v. 65, n.8, p. 653-655, 2011.

TURNER, Jerrold R.; KEEGAN, Anne. E. Mechanisms of governance in the project-based organization: the role of the broker and steward. European Management Journal, [s.l.], v. 19, n. 3, p. 254-267, 2001.

UNITED NATIONS (UN). Global health and foreign policy. Resolution A/67/L.36. Nova York: United Nations General Assembly, 2012. Disponível em: <http://www.un.org/ga/search/
view_doc.asp?symbol=A/67/L.36>. Acesso em: 31/08/2019.

VAN LERBERGHE, Wim. et al. When staff is underpaid: dealing with the individual coping strategies of health personnel. Bulletin of the World Health Organization, Genebra, v. 80, n. 7, p. 581-584, 2002.

VON BERTALANFFY, Ludwig. General system theory: foundations, development, applications. Revised edition. New York: George Braziller, 1969.

WORLD HEALTH ORGANIZATION (WHO). Health systems: improving performance. World Health Report 2000. Geneva: World Health Organization, 2000.

WORLD HEALTH ORGANIZATION (WHO). Integrated health services: what and why? Geneva: World Health Organization, 2008. Disponível em: <http://www.who.int/healthsystems/service_delivery_techbriefl.pdf $>$. Acesso em: 31/08/2019.

WORLD HEALTH ORGANIZATION (WHO). Increasing access to health workers in remote and rural areas through improved retention: global policy recommendations. Geneva: World Health Organization, 2010.

WORLD HEALTH ORGANIZATION (WHO). Global Strategy on Human Resources for Health: synthesis paper development. 2015. Disponível em: <https://www.medicusmundi.org/ contributions/reports/2014/how-to-address-health-workers-migration-in-the-global-strategy-on-human-resources-for-health $>$ Acesso em: 18/10/2015.

ZERE, Eyob et al. Technical efficiency of distrit hospitals: evidence from Namibia using data envelopment analysis. Cost Effetiveness and Resource Allocation, v. 4, n. 5, 2006. DOI: 10.1186/1478-7547-4-5 